After showing the ubiquity of the bacillus coli communis as an intestinal saprophyte, I must state my firm belief in auto-intoxic tion by that organism and explain the whole question by reference to certain unknown conditions of lowered body resistance culminating in what amounts to transmutation of a saprophyte into a parasite. Ptomaine poisoning may be regarded as an evidence of a similar process and therefore allied to a typical typhoid or paratyphoid fever, as may be seen from Lieutenant-Colonel Spencer's interesting comparative charts. In a private communication Lieutenant-Colonel Spencer points out that he uses an "abortive treatment" in these cases which consists in frequent flushing of the colon by large enemata of Condy's fluid and the administration of sulphate of quinine and sodium salicylate in alternate doses. He writes: "By this means I have never failed to bring the case to a favourable termination by the end of the second week." I have for several years been an advocate of colic lavage (izal) in dysenteric conditions ${ }^{7}$ but where the ulceration is above the ileo-cæcal valve this treatment would appear to be of little value.

Granting, then, that paratyphoid fever exists and that in India it appears to be commoner than in England one or two important questions arise which certainly cannot be answered at present. Is there, after all, a transmutation of different species of bacilli as was once claimed in the case of the bacillus anthracis and the hay bacillus? How does the bacillus coli communis attain its power of changing its saprophytic habits for parasitic ones? Is it as a result of lowered resistance of its host or certain unknown changes in man which allow auto-infection or is the power gained by the bacillus in its extra corporeal existence? The conditions of life vary so much in the tropics from those of the temperate zone that it is idle to seize upon one factor. Nevertheless, one may suggest the frequency with which human fæces pass through the bodies of other animals as a possible cause.

In England none of the higher animals feed on human excrement, but in India there are many-thus the pig, the dog, and certain carrion birds do so habitually-i.e., it is their staple article of food. Hares, partridges, peafowl, cattle, and buffaloes are very frequently foul feeders and any or all these latter are eaten by man or in the case of cattle their milk is consumed. It is a matter of common knowledge that many organisms undergo an exaltation in virulence by being passed throngh the bodies of animals and it is possible that in this way increased potentialities for evil are acquired by the typho-coli group. Enough has been written to show that another problem enters into Indian sanitation, the origin of paratyphoid variations. Are we, therefore, to go on looking upon the bacillus coli communis and its congeners as harmless organisms to be tolerated in pure water? Will it be necessary to inoculate our Indian drafts of soldiers with a polyvalent antityphoid vaccine like that being made polyvalent for the various micrococci of disease? Whatever answers will in the near future be given to these questions, one thing stands certain - the unity of typhoid fever can no longer be upheld.

My thanks are due to Dr. Row of Bombay for a demonstration of his specimens, methods, and literature, and to Lieutenant-Colonel Spencer for much help and the use of his published articles.

Bombay.

\section{INFECTIVE PURPURA.}

By S. ROBERT SOHOFIELD, M.B. LOND., M.R.C.S. ENG.

SEVERAL considerations have prompted me to place the following case of purpura on record. The remarkable features and the extreme gravity of the condition from which recovery eventually took place are in themselves sufficient justification for this, especially as reference to the literature on the subject shows our present knowledge to be very imperfect. Furthermore, I am anxious to call attention to the beneficial influence which the prolonged administration of quinine exerted on the clinical course of the illness,

7 Vide the Special Dysentery Number of the Indian Medical Gazette, July, 1905. for in regard to treatment also literature leaves much to be desired. The following is a short account of the case.

The patient, a married woman, aged 34 years, had been in poor state of health for several months, but had had no definite illness until about 36 hours before my first visit. The symptoms complained of were severe frontal headache and pains in the back and limbs. It was evening when she was first seen and the temperature was $104^{\circ} \mathrm{F}$. and the pulse was 124. A few small petechial extravasations were noticed and these were confined to the areas just above and below the knees. Six grains of salicylate of quinine and 15 grains of phenacetin divided into three cachets were given during the night with the result that the pains were eased and a rapid fall of temperature ensued, but this was accompanied by extreme restlessness and frequent attacks of vomiting. On the following mornirg the temperature was found to be subnormal but the pulse was still rapid (120). The signs of purpura were much more obvious and the area invaded became gradually extended until the following day when the limit appeared to be reached. The purpuric patchts varied in size, the largest being situated in front of the knees and about three and a half inches in diameter. There were large patches in the skin over each elbow, on the dorsal surface of the hands and of the joints of all the fingers but not the thumbs. The feet were similarly affected but to a lesser extent, the skin over the malleoli and the metacarpo-phalangeal joint of the great toes showing the largest patches. Similar extravasa tions were also noticed behind the spines of the scapulse and on the lower part of the back. There were numerous smaller patches on the dorsal surface of the limbs, the largest of these being nearest to the elbows and knees. The distribution suggested that the amount of extravasation was greater in the area of skin stretched over bony prominences and was promoted by the retarded circulation in these parts. The mucous membrane was not affected so seriously as the skin. There was slight hæmorrhage from the mouth; and the surface of the lips, mouth, and tongue was covered with a yellowish white slough and the mucous membrane of the nostrils and conjunctivæ was similarly affected. There was also a slight bloodstained discharge from the other mucous membranes, but during the whole course of the illness there did not appear to be any sign of internal hæmorrhage and the retina was also quite free. The area of the various patches had reached its maximum within 36 hours of the first appearance of purpura and no forther extravasations took place. The urine was examined at this stage and was found to contain albumin-about one-sixth. The patient continued to be very restless and collapsed and could not be induced to take even liquid food by the mouth. Rectal feeding was enployed and to the nutrient enemata salicylate of quinine and brandy were added. The restless. ness continued and it was only after successive doses of opium-trional and ammonium bromide having been found useless that any sleep was obtained. About the fifth day the mucous membranes of the mouth, tongue, and nostrils began to slough and repiration was greatly impeded. The nose and throat were frequently washed with boric lotion by means of a nasal douche and the fauces were painted with glycerine of borax. In the course of a few days necrotic patches of mucous membrane began to separate but there was no deep sloughing of these parts. The purpuric patches scattered over the trunk and limbs pursued the following course. The superficial layer of the skin became dry and the margin raised by an exudation at first serous and then purulent. In the smaller patches the skin could be peeled off in course of time, leaving a slightly pigmented surface. In the case of the larger patches, on the removal of the skin a smooth surface, dark brown in colour, was exposed. At first this was quite dry but soon pus begar to exude round the edges and when this necrotic portion became separated the tissue beneath was that of a sloughing sore. The depth of each slough varied directly with the size of the original patch. Thus, in the case of the elbows and knees the periosteum was exposed to a con siderable extent, and on the dorsum of the hand the sheaths of the extensor tendons were clearly visible. The back of each hand was practically one large slough. The inner surface of the great toes showed a similar condition. When the disease had lasted for about a month these sloughing surfaces were at their worst. They became gradually more healthy and began to granulate, and at the end of three months they were ail soundly healed. During the period when the sloughing was very extensive-for 
about six weeks-the general condition of the patient continued to be very serious. The temperature was irregular, varying from $101^{\circ}$ to $104^{\circ}$. There were frequent rigors, sometimes as many as three in the course of 24 hours. Towards the end of the second week the left ventricle became dilated, the apex beat being at one period an inch outside the nipple line. There was a well-marked systolic murmur, but the second sound remained clear. The albumin gradually disappeared from the urine and there was no trace of this after the first month. At the end of the sixth week the left leg became swollen and painful; this was evidently due to thrombosis. About this time there were signs of general improvement and the temperature gradually came down to normal. The pulse improved in quality and the left ventricle gained tone and the murmurs eventually disappeared. Three months after the onset the patient began to sit up in bed and a month later she was able to journey to the seaside. On her return six months later it was obvious that she had made an excellent recovery. She had gained greatly in weight and by the aid of massage and passive movements had recovered the full use of her limbs except that she could not completely close her hands owing to the con traction of the scars. This condition is now gradually improving.

Of the various forms of purpura that are described the case under consideration evidently belongs to the "infective" variety. The whole course of the illness and the behaviour of the purpuric areas admit of no other explanation as at all probable. What was the actual source of the infection it is impossible to say, for although the early symptoms suggested influenza there was no confirmatory evidence thit this was the case. As no other source of infection could be determined the precise cause of the patient's illness must remain in doubt. There were times when her condition was desperate-indeed, wellnigh hopeless - as may be gathered from the above account and the satisfactory termination of so grave an illness may, I think, be mainly ascribed to the influence of quinine. As mentioned above, the first drug employed was the salicylate of quinine. Two grains of this were given every four hours for the first ten days but with no obvious result. After this salicylate of soda was tried and then perchloride of iron, but these proved even less useful. During the third week, at the suggestion of Dr. H. P. Hawkins, who saw the patient in consultation with me, intramuscular injections of carbolic acid were employed. Five minims of a 5 per cent. solution were injected daily for six days. The object of this treatment was to stop the frequent rigors bu there was no obvious effect on the course of the illness. Had this form of treatment been instituted at an earlier stage of the disease a better result might have followed. At the end of the third week sulphate of quinine was prescribed. The dose was rapidly increased to four grains and this was given in an effervescing mixture every four hours. The drug was well tolerated by the patient and she continued to take 24 grains a day until the temperature had been normal for a fortnight, when the quinine was gradually discontinued. Under the influence of this treatment the rigors became less frequent and the general condition was much improved. In addition to the quinine, the other drugs employed were digitalis and strychnine; and, finally, iron, arsenic, and strychnine were given in combination in the form of a capsule.

The local treatment was naturally a matter of great importance, for there was much fear of septic absorption from the sloughing wounds. Frequent application of hot boric fomentations was first adopted but this plan of treatment had to be abandoned owing to the exhaustion caused by the constant disturbance of the patient. Antiphlogistine was substituted for the fomentations with marked advantage. This allowed the sloughs to be dressed only once a day and much exhaustion was thus avoided. Moreover, under the influence of this preparation the sloughing surfaces rapidly improved, the discharge from them became less offensive, and healthy granulations soon appeared, with the result that the wounds healed in the satisfactory way which has been described.

In conclusion, I wish to acknowledge my indebtedness to Dr. J. S. Risien Russell, who saw the patient on two occasions during the first week of her illness and who has kindly assisted $m e$ in the arrangement of these few notes of the case.

West Kensington, W.
40 CONSECUTIVE CASES OF FRACTURE OF THE PATELI.A TREATED BY WIRING.

By C. MANSELL MOULLIN, M.A., M.D. Oxon., F.R.O.S. ENG.,

SENIOR SURGEON TO, AXD LECTURER ON SURGERY AT, THE LONDON HUSPITAL.

IN the last 11 years I have had under my care 40 cases of racture of the patella which I have treated by some method of wiring. For the first year or two, until I felt certain that there would not be any ill result, $I$ adopted this method of treatment only in the case of young adults who were of sound constitution and to whom a good union was of great importance. Fractures in older and feebler patients were treated by splints in the old-fashioned way with the usual results. For the last eight years I have used the method of wiring in every case of this description that has come under my care, with one exception, no matter what was the size of the fragments, or whether they werd comminuted, or whether the patients appeared to be on the verge of delirium tremens. The one exception was a case of re-fracture in an enormously stout woman in whom no operation of any kind was advisable.

In the first 28 cases I tried most of the various methods of subcutaneous wiring that have been recommended from time to time. All of these, with the exception of Barker's, were soon dismissed as unsatisfactory. Barker's method, which consists in passing a wire vertically around both fragments, through the ligamentum patellæ below and the tendon of the quadriceps above, and twisting the ends together on the cutaneous surface, I practised until five years ago with what appeared to be a good result. In several instances $I$ thought I had secured bony union. In most the union, if fibrous, appeared to be so strong that it was not likely to yield. Only one or two were plainly bad. But when $\mathbf{I}$ began to make use of the Roentgen rays as a matter of routine, examining with them all fractures alike, it soon became evident that this method was not much better than the others. Even with the greatest care, making my assistant rub the two fragments together while I tightened the wire so as to insure their being in contact, the apposition was far from being what it ought to be. If the posterior edges of the broken surfaces were in contact the anterior never were and the gap between them was far wider than could be accounted for by the interposition of shreds of pre-patellar fascia. The fragments were tilted and manipulation did not prevent them from becoming tilted as the wire was tightened. The tilting could be diminished by the direct downward pressure of a firm pad upon the cutaneous surface, but this pressed the fascia down between the fragments and rendered bony union out of the question.

The last 12 cases I have treated by the open method with, $I$ believe, bony union in all but one, in which the tilting was not sufficiently corrected, and certainly with an enormous saving of time and inconvenience to the patient. The plan adopted has been the same in all. Operation is performed on the third or fourth day after the accident when the patient has become more or less reconciled to bed and the swelling of the joint has ceased to increase. A semicircular flap is raised from over the frigments and all the blood clot is carefully turned out. Each fragment is drilled in such a way that the wire does not project through the cartilaginous surfaces. A single stout silver wire is passed through and the ends are twisted together until the fragments are in exact apposition, cut short, and buried. No carbolic acid or other antiseptic is allowed to touch the interior of the joint. There is no occasion to wash it out, as the blood, being all coagulated, can be removed with ease by means of a scoop and forceps. The torn fascia on the cutaneous surface of the patella is then united with catgut and one or two catgut sutures are used to join together the aponeurosis on either side if it has been extensively lacerated. No drain is inserted but the angle of the cutaneous incision is left a little open in case there should be any oozing. No splint is used. Deep and superficial dressings are applied and the knee is firmly bandaged. On the third or fourth day the bandages and the superficial dressings are removed and are replaced by a lighter bandage, and the patient is encouraged to llex and to extend the limb 